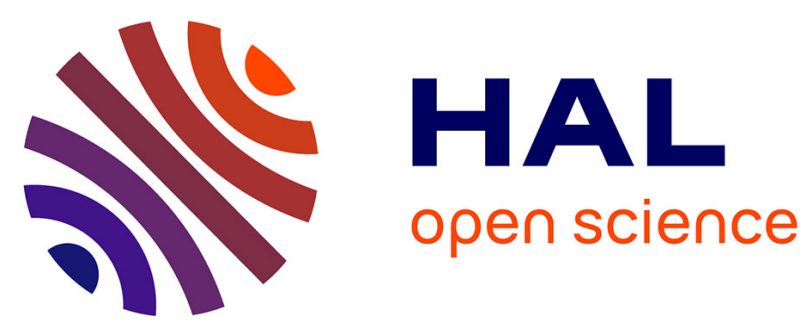

\title{
Grey matter density changes of structures involved in Posttraumatic Stress Disorder (PTSD) after recovery following Eye Movement Desensitization and Reprocessing (EMDR) therapy
}

Sarah Boukezzi, Myriam El Khoury-Malhame, Guillaume Auzias, Emmanuelle Reynaud, Pierre-François Rousseau, Emmanuel Richard, Xavier Zendjidjian, Jacques Roques, Nathalie Castelli, Nadia Correard, et al.

\section{- To cite this version:}

Sarah Boukezzi, Myriam El Khoury-Malhame, Guillaume Auzias, Emmanuelle Reynaud, PierreFrançois Rousseau, et al.. Grey matter density changes of structures involved in Posttraumatic Stress Disorder (PTSD) after recovery following Eye Movement Desensitization and Reprocessing (EMDR) therapy. Psychiatry Research: Neuroimaging, 2017, 266, pp.146-152. 10.1016/j.pscychresns.2017.06.009 . hal-02479828

\section{HAL Id: hal-02479828 \\ https://hal.science/hal-02479828}

Submitted on 6 Apr 2020

HAL is a multi-disciplinary open access archive for the deposit and dissemination of scientific research documents, whether they are published or not. The documents may come from teaching and research institutions in France or abroad, or from public or private research centers.
L'archive ouverte pluridisciplinaire HAL, est destinée au dépôt et à la diffusion de documents scientifiques de niveau recherche, publiés ou non, émanant des établissements d'enseignement et de recherche français ou étrangers, des laboratoires publics ou privés. 


\section{ACCEPTED MANUSCRIPT}

Grey matter density changes of structures involved in Posttraumatic Stress

Disorder (PTSD) after recovery following Eye Movement Desensitization and Reprocessing (EMDR) therapy

Sarah Boukezzi ${ }^{\mathrm{a} *}$, Myriam El-Khoury-Malhame ${ }^{\mathrm{b}}$, Guillaume Auzias, ${ }^{\mathrm{a}, \mathrm{c}}$, Emmanuelle Reynaud $^{\mathrm{a}}$, Pierre-François Rousseau, Emmanuel Richard ${ }^{\mathrm{d}}$, Xavier Zendjidjian ${ }^{\mathrm{d}}$, Jacques Roques $^{\mathrm{e}}$, Nathalie Castelli ${ }^{\mathrm{f}}$, Nadia Correard ${ }^{\mathrm{g}}$, Valérie Guyon ${ }^{\mathrm{d}}$, Caroline Gellato ${ }^{\mathrm{g}}$, Jean-Claude Samuelian $^{\mathrm{d}}$, Aida Cancel $^{\mathrm{a}, \mathrm{h}}$, Magali Comte ${ }^{\mathrm{a}}$, Marianne Latinus ${ }^{\mathrm{a}}$, Eric Guedj, ${ }^{\mathrm{a}, \mathrm{i}, \mathrm{j}}$, Stéphanie Khalfa ${ }^{\mathrm{a}}$.

${ }^{a}$ Institut de Neurosciences de la Timone, UMR 7289, Aix Marseille Université \& CNRS, Marseille, France

${ }^{\mathrm{b}}$ Lebanese American University, Faculty of Arts and Sciences, Byblos, Lebanon

${ }^{c}$ Laboratoire des Sciences de l'Information et des Systèmes, UMR 7296, Aix-Marseille Université \& CNRS, Marseille, France

d Assistance Publique des Hôpitaux de Marseille (APHM), Conception, CUMP, Marseille, France

${ }^{\text {e }}$ Centre de Traitement des Traumatismes Psychiques de Montpellier, Montpellier, France Institut Français d'EMDR, association EMDR, Paris, France

${ }^{\mathrm{g}}$ Assistance Publique des Hôpitaux de Marseille (APHM), Sainte Marguerite, Pôle de psychiatrie, Marseille, France

${ }^{\mathrm{h}}$ Centre Hospitalier Universitaire de Saint-Etienne, Pôle de Psychiatrie, Saint-Etienne, France

${ }^{\mathrm{i} A s s i s t a n c e}$ Publiques des Hôpitaux de Marseille (APHM), Timone, Service Central de Biophysique et Médecine Nucléaire, Marseille, France

${ }^{\mathrm{j}}$ Centre Européen de Recherche en Imagerie Médicale (CERIMED), Marseille, France 


\title{
ACCEPTED MANUSCRIPT
}

*Corresponding author: Sarah Boukezzi, M.S., Institut de Neurosciences de la Timone UMR 7289, CNRS-AMU Campus santé timone 27, Bd Jean Moulin 13385 Marseille cedex $5^{\mathrm{e}}$. Tel.: +33(0)4913240 83. sarah.boukezzi@univ-amu.fr

\begin{abstract}
Recovery of stress-induced structural alterations in Posttraumatic Stress Disorder (PTSD) remains largely unexplored. This study aimed to determine whether symptoms improvement is associated with GM density changes of brain structures involved in PTSD. Two groups of PTSD patients were involved in this study. The first group was treated with Eye Movement Desensitization and Reprocessing (EMDR) therapy and recovered from their symptoms (recovery group) $(\mathrm{n}=11)$; Patients were scanned prior to therapy (T1), one week (T2) and five months after the end of therapy (T3). The second group included patients which followed a supportive therapy and remained symptomatic (wait-list group) $(\mathrm{n}=7)$. They were scanned at three time-steps mimicking the same inter-scan intervals. T1-weighted images of anatomical structure were acquired using magnetic resonance imaging (MRI) prior to therapy (T1). Voxel-based morphometry (VBM) was used to characterize GM density evolution. GM density values showed a significant group-by-time interaction effect between $\mathrm{T} 1$ and $\mathrm{T} 3$ in prefrontal cortex areas. These interaction effects were driven by a GM density increase in the recovery group with respect to the wait-list group. Symptoms removal goes hand-in-hand with GM density enhancement of structures involved in emotional regulation.
\end{abstract}

\section{Key words:}

Posttraumatic Stress Disorder; Neuroanatomy; longitudinal MRI; EMDR therapy; recovery

\section{Introduction}

Posttraumatic Stress Disorder (PTSD) is a debilitating disorder characterized by repetition or revival, anxious hypervigilance, avoidance, and emotional numbing (Ursano et al., 2004). The neural correlates underlying PTSD symptomatology have garnered increasing 


\section{ACCEPTED MANUSCRIPT}

attention and findings posit marked anatomical key structures as the neural basis underlying this pathology. For instance structural neuroimaging findings in PTSD patients have consistently highlighted GM volume reductions in the medial prefrontal cortex (mPFC) (Rauch et al., 2003) and in the hippocampus (Bremner et al., 1997) as compared to healthy controls. Volumetric studies of the amygdala however remain inconclusive as some authors have reported smaller amygdala in PTSD patients than in healthy controls (Rogers et al., 2009), whereas others described no change (Yamasue et al., 2003) and others yet report an increased volume (Kuo et al., 2012). One recent meta-analysis has shown that PTSD was characterized by decreased volume in the hippocampus and the anterior cingulate cortex (ACC) when compared to trauma-exposed controls and unexposed controls (O'Doherty et al., 2015). PTSD patients were also found to have smaller amygdala than trauma-exposed controls, but no differences were found between patients and non-exposed controls (O’Doherty et al., 2015).

From a functional viewpoint, increased amygdala activity and diminished ventromedial PFC (vmPFC) activity seem to be the most robust finding in PTSD patients vs. healthy controls. One study accordingly depicted a model of PTSD in which cortical capacity to inhibit fear responses elicited by the amygdala would be altered (Etkin and Wager, 2007). Hippocampal findings show less consistency with both reports of exaggerated (Shin and Liberzon, 2010), and diminished activity (Bremner et al., 2003). As such, a new functional meta-analysis characterizes PTSD as involving a hyperactive amygdala and hypoactive medial prefrontal regions, additionally suggesting a hyperactive hippocampus (Patel et al., 2012).

In parallel with humans studies, findings from animal experiments suggest altogether that chronic stress induces dendritic atrophy in the mPFC (Cook and Wellman, 2004) and the hippocampus (McKittrick et al., 2000), whereas it induces a volumetric increase in the 


\section{ACCEPTED MANUSCRIPT}

amygdala (Vyas et al., 2003). Interestingly, after stress termination, recovery of stressinduced alterations in the neuronal architecture is observed in both the hippocampus and the mPFC, while persistent dendritic growth and spine formation is retained in the amygdala (Roozendaal et al., 2009).

Although to date neural and functional alterations in PTSD have been massively investigated; recovery of these functional and anatomical alterations remains largely understudied. As such, one study (Nardo et al., 2010) has shown that GM density was negatively correlated with trauma load in bilateral posterior cingulate, left anterior insula and right anterior parahippocampal gyrus. Further, GM volume increase was evidenced in the hippocampus of PTSD patients after medication (Vermetten et al., 2003) and Eye Movement Desensitization and Reprocessing (EMDR) therapy (Letizia et al., 2007). Moreover, an increased dorsolateral PFC (dlPFC) thickness was found after trauma recovery in disaster survivors compared to healthy controls (Lyoo et al., 2011). Yet to date, only one study has explored structural changes in the amygdala after recovery, revealing that left amygdala mean volume increased significantly after EMDR therapy (Laugharne et al., 2016). Functional decrease in amygdala activity was also reported after treatment in PTSD (Peres et al., 2011).

With the current scarcity of studies on GM density changes after stress recovery, and the discrepant results available in the literature related to such changes, no firm conclusions can be drawn as to the potential brain reorganization after recovery from trauma in PTSD patients. The aim of the present study was thus to determine whether GM density of brain structures involved in PTSD changes with symptoms improvement, as compared to GM density in PTSD with persisting symptoms. We addressed this issue using a Voxel-Based Morphometry (VBM) longitudinal study with two groups of PTSD patients. One group was treated with EMDR and subsequently recovered (recovery group) whereas the other, who followed only a supportive therapy, remained symptomatic (wait-list group). 


\section{ACCEPTED MANUSCRIPT}

To the best of our knowledge this is the first study to address changes in brain structures in PTSD immediately after symptoms removal and several months after. Based on the available literature defining brain regions involved in PTSD (Bremner et al., 2007; Francati et al., 2007; Wignall et al., 2004), as well as data from animal studies on stressinduced structural remodeling (Radley et al., 2008, 2005, 2004; Vyas et al., 2004), we hypothesized that GM density would increase in the PFC, the bilateral hippocampus and parahippocampal gyrus with symptoms amelioration. Moreover, in accordance with the functional decrease in amygdala activity after treatment (Peres et al., 2011), we expected GM density in the amygdala to decrease after recovery.

\section{Materials and methods}

\subsection{Participants}

A prospective, multi-center, non-randomized controlled clinical study compared two groups of patients suffering from PTSD. One group of patients (the recovery group) received EMDR therapy at the psychiatric pole of the "Conception Hospital" whereas the other group (the wait-list group) had only supportive therapy at the psychiatric pole of the "Sainte Marguerite Hospital" in Marseille, France. Patient's randomization was rendered unfeasible across the trauma centers mainly due to geographical distances. Diagnosis of PTSD was established according to the DSM-IV (Ursano et al., 2004). The Posttraumatic Checklist Scale (PCL-S) was used to assess the severity of PTSD symptoms along three dimensions (reexperiencing, avoidance and hyper-arousal symptoms) (Ventureyra et al., 2002).

Each group was scanned at three time points at the same fMRI center, in Marseille. Before each scan, participants were assessed with the DSM-IV and filled the PCL-S. 


\section{ACCEPTED MANUSCRIPT}

Participants of the recovery group were recruited among trauma-exposed victims by 2 psychiatrists at the psychiatric pole of the "Conception Hospital", in Marseille, France. This group comprised a total of 19 adult outpatients who met the DSM-IV criteria for PTSD after a single traumatic event with no previous history of neurologic or psychiatric disorders. Once the diagnosis was established, participants were scanned at baseline (T1). Participants in this group were treated with EMDR. EMDR is an eight-step standardized psychotherapy validated by the American Psychiatric Association (APA) reports published in 2004, as a first line treatment for PTSD. Based on an information processing model, EMDR founder Shapiro et al., (2002) stipulate it includes associations of cognitive, emotional, and physical assessments of actual distress to traumatic scenery, as well as imaginal exposure. As the patient is asked to visualize the most salient aspect of a traumatic memory, the therapist induces bilateral alternate stimulations (by means of ocular, sensory-motor, or auditory stimulation) (Shapiro, 1989). EMDR is an effective and rapid therapy with stable outcome reported in a 35 -month follow-up study (Högberg et al., 2008). Patients were treated by one of 4 therapists trained by the French institute of EMDR. All therapists used ocular bilateral stimulations. There was no fixed number of sessions. One-hour sessions were planned every 7 to 15 days according to the availabilities of patients and therapists. The treatment was considered successful and complete when patients had no more symptoms, reported no more feelings of distress when thinking about their trauma and no longer met PTSD criteria according to DSM-IV. They also had to score below cut-off for psychopathology on the PCL-S scale. PTSD patients required an average of $2.5(\mathrm{SD}=1.3)$ treatment sessions, ranging from 1 to 5 sessions. Individual sessions lasted around $1 \mathrm{~h}$ and so the total mean time of EMDR therapy was $2 \mathrm{~h} 30$ per patient on average. Symptom-free patients were then scanned for the second time one week after the symptoms removal (T2) and then the third and last time five months after T2 (T3). 


\section{ACCEPTED MANUSCRIPT}

Seven participants dropped out after recovery, between T2 and T3, and one patient's data was excluded from the analysis because of excessive head motion during image acquisition. Hence, the final recovery group included 11 adult outpatients (with 4 females). In its final makeup the recovery group included 1 patient on hypnotic medication, 2 patients on antidepressant, and 1 patient with a combination of anxiolytic, antidepressant and hypnotic. Eight participants had comorbid major depressive disorder (MDD) or other anxiety disorders. Characteristics of participants are described in Table 1.

PTSD participants of the wait-list group were recruited among trauma-exposed victims by 2 other psychiatrists at the psychiatric pole of the "Sainte-Marguerite Hospital" in Marseille, France. Patients of this group followed a supportive therapy only, and retained their symptoms throughout the study. The supportive therapy is a non-specified form of therapy, with limited goals, centered on the adaptation of patients. This psychotherapy does not share any of the active components of EMDR but rather combines supportive talk and empathy with suggestive resources (Rockland, 1989). It was administered by psychologists and psychiatrists of the "Sainte-Marguerite Hospital". Patients of wait-list group were scanned at three time points T1, T2 and T3, with inter-scan intervals similar to the recovery group. At the end of the study, participants in this group were given the option to enroll in EMDR therapy. The waitlist group initially included 16 adult PTSD outpatients who retained their PTSD diagnosis by T3. Between T2 and T3, 5 of them gave up on the study and 4 others were excluded from the final analyses because of excessive head motion during image acquisition. Hence, the final wait-list group included 7 outpatients (with 4 females). One patient in this group was on a stable regimen of antidepressant, 1 took both antidepressant and anxiolytic, 1 had anxiolytic only and 2 patients had a combination of anxiolytic, antidepressant and hypnotic. Two participants had comorbid MDD and another anxiety disorder, and 4 participants had only one comorbid anxiety disorder. 


\section{ACCEPTED MANUSCRIPT}

The study was reviewed and approved by the local ethics committee (CPP South Mediterranean 2). All procedures were in accordance with the ethical standards of the committee on human experimentation (institutional and national) and with the Helsinki Declaration of 1975, and the applicable revisions at the time of the investigation. Informed consent was obtained from all patients for being included in the study. The registry name of this study was "Posttraumatic Stress Disorder and Neural Mechanisms involved in Its Treatment" and the trial registration number was 2009-A00193-54.

Statistical analyses of demographic data were performed using SPSS (v18.0, SPSS Inc., Chicago, IL) and are depicted in Table 1. Demographic differences between groups were assessed with independent t-test at T1. Changes of PCL-S scores were assessed with a twoway repeated measure ANOVA with Group (recovery / wait-list) as a between factor and Time (T1, T2, T3) as a within-subject factor. Post-hoc analyses were performed in order to detect changes in PCL-S scores between scans. Bonferroni correction was applied to correct for multiple-testing.

As detailed in Table 1, the two groups were matched for education, age, PCL-S scores at $\mathrm{T} 1$ and interscan intervals (T2-T1, T3-T1 and T3-T2). The duration of illness between the two groups was not significant $(\mathrm{p}=0.051)$. 


\section{ACCEPTED MANUSCRIPT}

\subsection{MRI acquisition parameters}

For the T1 (baseline), T2 and T3 scans, the structural brain data was acquired on a 3-T MEDSPEC 30/80 AVANCE imager (Bruker, Ettlingen, Germany) at the fMRI center of the Timone hospital in Marseille, France. High-resolution T1-weighted images were acquired using the following parameters: sagittal orientation, MPRAGE sequence, echo time $=4.42$ $\mathrm{ms}$, repetition time $=9.4 \mathrm{~ms}$, inversion time $=800 \mathrm{~ms}$, flip angle $=30^{\circ}$, matrix $=$ $256 * 256 * 180$, Voxel size $=1 * 1 * 1 \mathrm{~mm}^{3}$. The scanning time for the MPRAGE sequence was 15 minutes. The total scanning time for each participant was 45 minutes.

\subsection{Voxel-Based Morphometry (VBM) Analysis}

VBM analyses were performed with the VBM8 toolbox (http://dbm.neuro.unijena.de/vbm/) for SPM8 (http://www.fil.ion.ucl.ac.uk/spm). VBM is an unbiased, semiautomated technique that allows the characterization of regional differences across the whole brain (Mechelli et al., 2005). It is a recent and promising neuroimaging technique that allows the investigation of focal differences in brain anatomy (Nardo et al., 2010). It was used to characterize the differences in regional brain tissue concentration.

Standard routines and default parameters of the VBM8 toolbox were applied for the main phases of the preprocessing and statistical analyses.

Individual preprocessing steps were replicated as follows. First, the baseline and follow-up images of each subject were realigned with SPM8. This step aims to improve the quality of the normalization and segmentation steps in the pretreated images. Images were centered by laying the origin of axes on the anterior commissure in order to minimize potential normalization problems and to reduce magnetic field inhomogeneities. The mean of these realigned images was then calculated and used as a reference image in a subsequent 


\section{ACCEPTED MANUSCRIPT}

realignment. The realigned images were corrected for inhomogeneities of the signal, with regard to the reference mean image. In the follow-up scans, the mean T1 image was segmented. Normalization parameters, estimated from the segmentation of the mean image, were then used to segment the bias-corrected images. Dartel deformations were then applied to normalize the segmented images into the MNI template (Montreal Neurological Institute). Finally, the resulting normalized segmented images were again realigned with one another. Unmodulated VBM identified differences in the relative concentration or density of grey or white matter (i.e., the proportion of grey or white matter relative to other tissue types within a region) (Mechelli et al., 2005). Once preprocessing was complete, we checked the quality of the images and removed unusual ones. Images were smoothed with an $8 \mathrm{~mm}$ FWHM (FullWidth Half-Maximum) isotropic Gaussian filter and included in the GM analysis with a flexible factorial design.

Smoothed GM images were thresholded at a value of 0.1 to exclude any remaining non-GM tissue and entered in a flexible factorial design with Group (recovery / wait-list) and Time (T1, T2, T3) as categorical factors. To restrain the analysis to GM density a mask of GM was defined using the Automated Anatomical Labeling atlas (Tzourio-Mazoyer et al., 2002) implemented in the WFU PickAtlas toolbox (Maldjian et al., 2003).

We investigated potential difference between the two groups in the evolution of GM density across the three time points. To do so, we built three contrasts corresponding to the group-by-time interaction effects: 1) recovery group (T2-T1) - wait-list group (T2-T1); 2) recovery group (T3-T2) - wait-list group (T3-T2) and 3) recovery group (T3-T1) - wait-list group (T3-T1). The resulting statistic maps were corrected for multiple comparisons with a $\mathrm{p}$ $<0.05$ family-wise error cluster-extent threshold (Bennett et al., 2009), using a primary statistical threshold at the voxel-level set at $\mathrm{p}<0.001$. GM density was then extracted in each significant cluster for each subject and time step point using SPM functions (spm_vol, and 
spm_read_vols). We then plotted the GM density mean difference of each cluster against time point in each group.

\section{Results}

\subsection{Participants' characteristics}

PCL-S scores at each time point are given in Table 1. As previously noted, PCL-S scores were matched at T1. In the wait-list group, PCL-S scores did not change between T1 and T2 $(\mathrm{p}=1.000)$ and between $\mathrm{T} 1$ and $\mathrm{T} 3(\mathrm{p}=1.000)$, while it significantly decreased in the recovery group between $\mathrm{T} 1$ and $\mathrm{T} 2(\mathrm{p}<0.001)$ and between $\mathrm{T} 1$ and $\mathrm{T} 3(\mathrm{p}<0.001)$. In the recovery group, EMDR was effective for all patients. Additionally, we observed a higher PCL-S score in the wait-list group than in the recovery group at T2 $(\mathrm{p}<0.001)$, and T3 $(\mathrm{p}<$ $0.001)$, with a significant group-by-time interaction $(\mathrm{F}(2,32)=25.34 ; \mathrm{p}<0.001)$.

\subsection{Longitudinal VBM results}

The results of the VBM analyses are illustrated in Fig 1 and summarized in Table 2. We found no significant group-by-time interactions for the contrast (recovery group (T3-T2) wait-list group (T3-T2)) and for the contrast (recovery group (T2-T1) - wait-list group (T2T1)). We found no significant group-by-time interaction in the amygdala, in the hippocampus, or the parahippocampal gyrus. The group-by-time interaction for the contrast (recovery group (T3-T1) - wait-list group (T3-T1)) was significant in four clusters in the frontal lobe (Fig 1 left). A first cluster was located in the right inferior frontal gyrus, including the dorsolateral prefrontal cortex and the orbitofrontal cortex (BA 46, 9, 10) (Fig 1A). A second significant cluster was found in the medial PFC (Fig 1B) comprising the dorsal ACC (BA 24, BA 32) 


\section{ACCEPTED MANUSCRIPT}

(Fig 1B). A third significant cluster was observed in the right middle frontal gyrus (BA 6)

(Fig 1C). Finally the fourth was situated in the bilateral mPFC (BA 9) (Fig 1D). In all 4 clusters, group-by-time interaction effects were driven by a GM density increase with time in the recovery group as compared to the wait-list group, as depicted by plots of GM density evolution in Fig 1 (right).

Figure 1. The significant clusters resulting from the VBM analysis $\left(\mathrm{P}_{\text {cluster-level }}<0.05\right.$ after FWE correction).

The significant clusters resulting from the VBM analysis $\left(\mathrm{P}_{\text {cluster-level }}<0.05\right.$ after FWE correction). Three orthogonal views are shown on the left; corresponding plots of GM density evolution for each cluster are shown on the right. Significant cluster for the interaction contrast: recovery group (T3-T1) - wait-list group (T3-T1). A: Inferior frontal gyrus (dlPFC+OFC); B: Anterior cingulate cortex (BA 24/32); C: Middle frontal gyrus; D: Medial PFC (BA 9).

\section{Discussion}

The aim of this study was to assess whether GM density in brain structures altered in PTSD changes with symptoms improvement. This study seems to provide evidence of significant group-by-time interactions driven by GM density increases post-EMDR, mostly in PFC structures.

To the best of our knowledge, this is the first VBM study suggesting that GM density increases in the PFC with PTSD symptoms improvement. The cellular and molecular mechanisms underlying GM density increase in humans have yet to be elucidated to establish whether these changes are the result of an enhancement of dendritic spine density, dendritic length and branching complexity, or the expression of spine- and synapse-related proteins as in animals (Gross, 2000). Whatever the underlying mechanism, this GM density enhancement 


\section{ACCEPTED MANUSCRIPT}

parallels existing data in animals; thereby suggesting a potential reversibility of stress-induced effects in the PFC (Roozendaal et al., 2009).

PTSD is mostly characterized by severe deficits in emotion regulation processes and is marked by emotional hyper-reactivity to trauma-related cues (Ursano et al., 2004). Under normal conditions, the top-down regulation of emotions, specifically the regulation of negative emotions and subsequent behaviors, is known to involve the mPFC and the IFG, including the dlPFC and the OFC (Davidson, 2000). These prefrontal regions have consistently shown altered activity and decreased volumes in PTSD. Herein we show significantly increased GM density in these same prefrontal regions after symptoms improvement by EMDR. As anatomical structure may bi-directionally drive and underlie function, one putative explanation of symptom removal by EMDR could encompass the restoration of prefrontal volume after therapy, potentially countering the initial decrease in these regions activity in PTSD. This would thereby reflect patients' improved ability to better deal with and control their negative emotions.

The present results parallel a previous study in which GM lower density in limbic and paralimbic cortices was found to be associated with PTSD diagnosis, trauma load, and EMDR treatment outcome (Nardo et al., 2010). Moreover, our results also parallel previous functional findings reporting successful restoration of prefrontal activity post-EMDR in PTSD, with significantly increased perfusion in the left frontal gyrus (Lansing et al., 2005), increased cerebral blood flow in the bilateral dlPFC (Oh and Choi, 2007), and increased activity in the mPFC, including the ACC (Levin et al., 1999). However, likely due to methodological differences with our experiment, other studies with larger sample size have shown that in patients suffering from PTSD, normalization of a tracer distribution in the perilimbic cortex occurred after successful EMDR therapy as compared to trauma exposed controls (Pagani et al., 2007). Moreover, it has been shown that GM lower density in limbic 


\section{ACCEPTED MANUSCRIPT}

and paralimbic cortices was associated to PTSD diagnosis, trauma load, and EMDR outcome (Nardo et al., 2010).

Furthermore, the trademark of PTSD's emotional dysfunctions is an exaggerated response to fear, along with an inability to inhibit it (Charney, 2004). The prevailing hypothesis puts the hyperactive amygdala at the core of the disorder alongside a downregulated $\mathrm{mPFC}$, ultimately failing to inhibit the limbic triggers (Hariri et al., 2000). Despite no changes found in the amygdala after symptom removal, significant GM density enhancement of the mPFC (BA 9, 24, 32) in the recovery group could unveil more potentiated mPFC that would become more efficient in regulating amygdala activity, ultimately driving fear extinction circuitry. Further longitudinal functional neuroimaging studies should verify that this GM density increase in prefrontal structures correlates with a restoration of central mechanisms involved in fear processing.

Also, this study is the first of its kind to investigate differential impact of short and long term therapeutic benefits of symptoms improvement on brain structures in PTSD. We only found a between group difference of GM density evolution between the first and the third scan, but not between the first and the second scan or between the second and the third scan. This suggests that neuroanatomical changes do not occur or at least cannot be measured immediately after symptom improvement and require several months for stabilization. Despite the immediacy of the symptoms improvement, morphological PFC changes do not occur as soon as symptoms disappearance but several months after. Animal studies have shown that morphological changes after antidepressant administration are observable immediately after taking the drug, but its effect on brain plasticity lasts only as long as medication is administered (Burgdorf et al., 2015). Although PTSD symptoms had disappeared by T2, and were non existent at T3, GM density increase was only found between T1 and T3. This might suggest that the effect on the brain structures of EMDR psychotherapy, 


\section{ACCEPTED MANUSCRIPT}

would take more time to initiate and potentiate than pharmacotherapy, but anatomical changes induced thereafter would last longer.

Contrary to our initial hypotheses, we found no significant group-by-time interaction effects in the amygdala, the hippocampus, or the parahippocampal gyrus. Interestingly, when the conservative cluster-level FWE correction was not applied, a significant group-by-time interaction emerged for the T3-T1 interval at the voxel level $(\mathrm{p}<0.001$ uncorrected) in two clusters including the bilateral parahippocampal gyrus, the hippocampus, and the amygdala. Accordingly, this lack of result in limbic structures could be accounted for by the reduced statistical power related to the small sample size in this study. Another possible explanation could relate to methodological considerations. We have indeed proceeded with a longitudinal pipeline using the standard routines and default parameters of the VBM8 toolbox. Images obtained at the end of the preprocessing were unmodulated. Unmodulated VBM identified differences in the relative concentration or density of GM (i.e., the proportion of GM relative to other tissue types within a region), whereas modulated images identified GM volumes. One previous study reported differences between analyses of GM concentration and GM volumes (Good et al., 2001), especially in hippocampal regions (Keller et al., 2004). Therefore, using the GM volume analyses instead of GM density may have yielded changes in others regions than previously reported in our study, such as, most likely the hippocampus and the amygdala.

The non-randomized design limits to some extent the interpretation of the group by time GM density changes. Moreover, the small sample size prevents the elaboration of a correlation between PTSD symptoms decrease and GM density increase in the recovery group. In spite of such limitation, our results suggest an association between GM density and EMDR therapy outcome, a finding with valuable clinical implications in PTSD treatment planning. Additional studies with larger groups of patients would be needed to replicate and 
further test for correlations between GM density changes and EMDR therapy outcome. Moreover, some of the patients were on stable medical regimen of antidepressants and/or anxiolytics and had other comorbid anxiety and/or mood disorders, all of which could potentially affect brain structures. Even though the duration of illness between the two groups was not significantly different, the effect size associated with this difference was large: Cohen's d effect size $d=0.8495 \%$ CI: $-0.39-105.96$. This indicates that the duration of illness between the recovery and the wait-list groups could introduce a confounding variable. Further studies including matched duration of illness between the groups would be recommended. Finally, for some significant clusters, at baseline, GM density in the recovery group was lower than for the wait-list group. This might be due to the heterogeneity of these two groups related to the type of trauma and the duration of illness since trauma. Yet, the aim of the study was to compare GM density evolution between the two groups and not merely to compare GM density between the two groups at each time point. It is thus noteworthy that despite these limitations, the validity of our results is strongly supported by the correction for multiple comparisons with a $\mathrm{p}<0.005$ family-wise error cluster-extent threshold.

This work contributes to a better understanding of biological brain mechanisms underlying PTSD symptoms recovery. It parallels animals' results on stress recovery. It also helps promoting the use of psychotherapies such as EMDR as early as possible after traumatic events to prevent further worsening of patients' symptomatology and structural alterations in the brain. 


\section{ACCEPTED MANUSCRIPT}

\section{Financial disclosures/conflict of interest}

The authors report no competing interests

\section{Funding}

This research was partially supported by a grant from the Direction Générale de l'Armement (DGA) awarded to Sarah Boukezzi, by a grant from the APHM (Public Assistance of Hospitals in Marseille), and by a grant from the EMDR Europe Association awarded to Stéphanie Khalfa.

\section{Acknowledgments}

We gratefully acknowledge all the PTSD participants for their time and involvement. We thank Bruno Nazarian and Jean-Luc Anton for their precious technical support and assistance with data collection.

Table 1. Mean and Standard deviation (SD) for the demographical and clinical characteristics of participants

\begin{tabular}{llll}
\hline Characteristics & Recovery group & Wait-list group & $\mathrm{p}$ \\
& $\mathrm{n}=11$ & $\mathrm{n}=7$ & \\
\hline Gender, Male/Female & $7 / 4$ & $4 / 3$ & 0.766 \\
Age, years & $34.9(10.0)$ & $34.5(8.1)$ & 0.926 \\
Education, years & $6.8(2.6)$ & $7.6(2.3)$ & 0.541 \\
Duration of illness, months & $57.6(79.1)$ & $4.8(2.8)$ & 0.051 \\
Trauma type & & & \\
Agressions, $\mathrm{n}$ & 4 & 6 &
\end{tabular}




\begin{tabular}{|c|c|c|c|}
\hline \multicolumn{4}{|c|}{ ACCEPTED MANUSCRIPT } \\
\hline road accidents, $\mathrm{n}$ & 3 & 1 & \\
\hline armed robberies, $\mathrm{n}$ & 1 & 0 & \\
\hline work-related accident, $\mathrm{n}$ & 1 & 0 & \\
\hline witness of a suicide, $\mathrm{n}$ & 1 & 0 & \\
\hline Torture, $\mathrm{n}$ & 1 & 0 & \\
\hline \multicolumn{4}{|c|}{ Interscan Interval, months } \\
\hline T2-T1 & $1.9(1.6)$ & $2.5(2.8)$ & 0.559 \\
\hline T3-T1 & $7.3(3.2)$ & $10.3(4.8)$ & 0.129 \\
\hline T3-T2 & $5.4(2.0)$ & $7.8(3.8)$ & 0.099 \\
\hline \multicolumn{4}{|l|}{ PCL-S scores } \\
\hline PCL-S T1 & $54.82(8.90)$ & $63.79(14.55)$ & 1.000 \\
\hline PCL-S T2 & $23.91(3.94)$ & $61.64(14.97)$ & $<0.001$ \\
\hline PCL-S T3 & $22.73(4.27)$ & $58.14(12.56)$ & $<0.001$ \\
\hline
\end{tabular}

PCL-S: Posttraumatic Checklist Scale; T1: Time 1; T2: Time 2; T3: Time 3

Table 2. VBM statistics for the interaction contrast "recovery group (T3-T1) - wait-list group (T3-T1)" after FWE cluster-correction for multiple comparisons.

\begin{tabular}{|c|c|c|c|c|c|c|c|}
\hline \multirow[b]{2}{*}{ Figure 1} & \multirow[b]{2}{*}{ Region and contrast } & \multirow[b]{2}{*}{ side } & \multirow[b]{2}{*}{ BA } & \multirow{2}{*}{\multicolumn{2}{|c|}{$\begin{array}{c}\text { MNI } \\
\text { coordinates } \\
\text { x y z }\end{array}$}} & \multirow[b]{2}{*}{ Z } & \multirow[b]{2}{*}{$\mathrm{p}$} \\
\hline & & & & & & & \\
\hline & recovery group (T3-T1) - wait-list g & & & & & & \\
\hline A & Inferior frontal gyrus (dlPFC, OFC) & $\mathrm{R}$ & $46 / 9 / 10$ & 1688 & 484015 & 4.57 & $<0.001$ \\
\hline B & Anterior Cingulate Cortex & $\mathrm{R} / \mathrm{L}$ & $24 / 32$ & 868 & -4643 & 4.47 & $<0.001$ \\
\hline $\mathrm{C}$ & Middle frontal gyrus & $\mathrm{R}$ & 6 & 562 & 42554 & 4.18 & $<0.001$ \\
\hline $\mathrm{D}$ & Medial PFC & $\mathrm{R} / \mathrm{L}$ & 9 & 812 & -24237 & 4.60 & $<0.001$ \\
\hline
\end{tabular}

Coordinates are given in MNI space. $\mathrm{p}=$ corrected $\mathrm{p}$-value; $\mathrm{k}=$ cluster size; $\mathrm{BA}=$ Brodmann area. dlPFC: dorsolateral prefrontal cortex; OFC: Orbitofrontal cortex. 


\section{References}

Bennett, C.M., Wolford, G.L., Miller, M.B., 2009. The principled control of false positives in neuroimaging. Soc. Cogn. Affect. Neurosci. 4, 417-422. doi:10.1093/scan/nsp053

Bremner, J.D., Elzinga, B., Schmahl, C., Vermetten, E., 2008. Structural and functional plasticity of the human brain in posttraumatic stress disorder. Prog Brain Res.167, 171186.doi:10.1016/S0079-6123(07)67012-5

Bremner, J.D., Randall, P., Vermetten, E., Staib, L., Bronen, R. A, Mazure, C., Capelli, S., McCarthy, G., Innis, R.B., Charney, D.S., 1997. Magnetic resonance imaging-based measurement of hippocampal volume in posttraumatic stress disorder related to childhood physical and sexual abuse--a preliminary report. Biol. Psychiatry 41, 23-32.

Bremner, J.D., Vythilingam, M., Vermetten, E., Southwick, S.M., McGlashan, T., Nazeer, A., Khan, S., Vaccarino, L.V., Soufer, R., Garg, P.K., Ng, C.K., Staib, L.H., Duncan, J.S., Charney, D.S., 2003. MRI and PET study of deficits in hippocampal structure and function in women with childhood sexual abuse and posttraumatic stress disorder. Am. J. Psychiatry 160, 924-932. doi:10.1176/appi.ajp.160.5.924

Burgdorf, J., Zhang, X. -l., Weiss, C., Gross, A., Boikess, S.R., Kroes, R.A., Khan, M.A., Burch, R.M., Rex, C.S., Disterhoft, J.F., Stanton, P.K., Moskal, J.R., 2015. The longlasting antidepressant effects of rapastinel (GLYX-13) are associated with a metaplasticity process in the medial prefrontal cortex and hippocampus. Neuroscience 308, 202-211. doi:10.1016/j.neuroscience.2015.09.004

Charney, D.S., 2004. Psychobiological mechanisms of resilience and vulnerability: implications for successful adaptation to extreme stress. Am. J. Psychiatry 161, 195-216. doi:10.1176/appi.ajp.161.2.195

Cook, S.C., Wellman, C.L., 2004. Chronic stress alters dendritic morphology in rat medial prefrontal cortex. J. Neurobiol. 60, 236-248. doi:10.1002/neu.20025

Davidson, R.J., 2000. Dysfunction in the neural circuitry of emotion regulation--a possible prelude to violence. Science. 289 (5479), 591-594. doi:10.1126/science.289.5479.591

Etkin, A., Wager, T.D., 2007. Functional neuroimaging of anxiety: a meta-analysis of emotional processing in PTSD, social anxiety disorder, and specific phobia. Am. J. Psychiatry 164, 1476-1488. doi:10.1176/appi.ajp.2007.07030504

Francati, V., Vermetten, E., Bremner, J.D., 2007. Functional neuroimaging studies in posttraumatic stress disorder: review of current methods and findings. Depress. Anxiety 24, 202-218. doi:10.1002/da.20208

Good, C.D., Johnsrude, I.S., Ashburner, J., Henson, R.N.A., Friston, K.J., Frackowiak, R.S.J., 2001. A Voxel-Based Morphometric Study of Ageing in 465 Normal Adult Human Brains. Neuroimage 14, 21-36. doi:10.1006/nimg.2001.0786

Gross, C.G., 2000. Neurogenesis in the adult brain: death of a dogma. Nat. Rev. Neurosci. 1, 67-73. doi:10.1038/35036235

Hariri, A.R., Bookheimer, S.Y., Mazziotta, J.C., 2000. Modulating emotional responses: effects of a neocortical network on the limbic system. Neuroreport 11, 43-48. 


\section{ACCEPTED MANUSCRIPT}

Högberg, G., Pagani, M., Sundin, Ö., Soares, J., Åberg-Wistedt, A., Tärnell, B., Hällström, T., 2008. Treatment of post-traumatic stress disorder with eye movement desensitization and reprocessing: Outcome is stable in 35-month follow-up. Psychiatry Res. 159, 101-108. doi:10.1016/j.psychres.2007.10.019

Keller, S.S., Wilke, M., Wieshmann, U.C., Sluming, V.A., Roberts, N., 2004. Comparison of standard and optimized voxel-based morphometry for analysis of brain changes associated with temporal lobe epilepsy. Neuroimage 23, 860-868. doi:10.1016/j.neuroimage.2004.07.030

Kuo, J.R., Kaloupek, D.G., Woodward, S.H., 2012. Amygdala volume in combat-exposed veterans with and without posttraumatic stress disorder: a cross-sectional study. Arch. Gen. Psychiatry 69, 1080-1086. doi:10.1001/archgenpsychiatry.2012.73

Lansing, K., Amen, D.G., Hanks, C., Rudy, L., 2005. High-resolution brain SPECT imaging and eye movement desensitization and reprocessing in police officers with PTSD. J. Neuropsychiatry Clin. Neurosci. 17, 526-532. doi:10.1176/jnp.17.4.526

Laugharne, J., Kullack, C., Lee, C.W., McGuire, T., Brockman, S., Drummond, P.D., Starkstein, S., 2016. Amygdala volumetric change following psychotherapy for posttraumatic stress disorder. J. Neuropsychiatry Clin. Neurosci. appineuropsych16010006-appineuropsych16010006.

doi:10.1176/appi.neuropsych.16010006

Letizia, B., Andrea, F., Paolo, C., 2007. Neuroanatomical changes after eye movement desensitization and reprocessing (EMDR) treatment in posttraumatic stress disorder. J. Neuropsychiatry Clin. Neurosci. 19, 475-476. doi:10.1176/jnp.2007.19.4.475

Levin, P., Lazrove, S., van der Kolk, B., 1999. What psychological testing and neuroimaging tell us about the treatment of Posttraumatic Stress Disorder by Eye Movement Desensitization and Reprocessing. J. Anxiety Disord. 13, 159-172. doi:10.1176/jnp.2007.19.4.475

Lyoo, I.K., Kim, J.E., Yoon, S.J., Hwang, J., Bae, S., Kim, D.J., 2011. The neurobiological role of the dorsolateral prefrontal cortex in recovery from trauma. Longitudinal brain imaging study among survivors of the South Korean subway disaster. Arch. Gen. Psychiatry 68, 701-713. doi:10.1001/archgenpsychiatry.2011.70

Maldjian, J. A., Laurienti, P.J., Kraft, R. A., Burdette, J.H., 2003. An automated method for neuroanatomic and cytoarchitectonic atlas-based interrogation of fMRI data sets. Neuroimage 19, 1233-1239.

McKittrick, C.R., Magariños, A.M., Blanchard, D.C., Blanchard, R.J., McEwen, B.S., Sakai, R.R., 2000. Chronic social stress reduces dendritic arbors in CA3 of hippocampus and decreases binding to serotonin transporter sites. Synapse 36, 85-94. doi:10.1002/(SICI)1098-2396(200005)36:2<85::AID-SYN1>3.0.CO;2-Y

Mechelli, A., Friston, K.J., Frackowiak, R.S., Price, C.J., 2005. Structural covariance in the human cortex. J. Neurosci. 25, 8303-8310. doi:10.1523/JNEUROSCI.0357-05.2005

Nardo, D., Högberg, G., Looi, J.C.L., Larsson, S., Hällström, T., Pagani, M., 2010. Gray matter density in limbic and paralimbic cortices is associated with trauma load and EMDR outcome in PTSD patients. J. Psychiatr. Res. 44, 477-485. 
doi:10.1016/j.jpsychires.2009.10.014

O’Doherty, D.C.M., Chitty, K.M., Saddiqui, S., Bennett, M.R., Lagopoulos, J., 2015. A systematic review and meta-analysis of magnetic resonance imaging measurement of structural volumes in posttraumatic stress disorder. Psychiatry Res. 232, 1-33. doi:10.1016/j.pscychresns.2015.01.002

Oh, D.-H., Choi, J., 2007. Changes in the regional cerebral perfusion after eye movement desensitization and reprocessing: A SPECT study of two cases. J. EMDR Pract. Res. 1, 24-30. doi:10.1891/1933-3196.1.1.24

Pagani, M., Högberg, G., Salmaso, D., Nardo, D., Sundin, O., Jonsson, C., Soares, J., AbergWistedt, A., Jacobsson, H., Larsson, S.A., Hällström, T., 2007. Effects of EMDR psychotherapy on 99mTc-HMPAO distribution in occupation-related post-traumatic stress disorder. Nucl. Med. Commun. 28, 757-765. doi:10.1097/MNM.0b013e3282742035

Patel, R., Spreng, R.N., Shin, L.M., Girard, T.A., 2012. Neurocircuitry models of posttraumatic stress disorder and beyond: a meta-analysis of functional neuroimaging studies. Neurosci. Biobehav. Rev. 36, 2130-2142. doi:10.1016/j.neubiorev.2012.06.003

Peres, J.F.P., Foerster, B., Santana, L.G., Fereira, M.D., Nasello, A.G., Savoia, M., MoreiraAlmeida, A., Lederman, H., 2011. Police officers under attack: resilience implications of an fMRI study. J. Psychiatr. Res. 45, 727-734. doi:10.1016/j.jpsychires.2010.11.004

Radley, J.J., Rocher, A.B., Janssen, W.G.M., Hof, P.R., McEwen, B.S., Morrison, J.H., 2005. Reversibility of apical dendritic retraction in the rat medial prefrontal cortex following repeated stress. Exp. Neurol. 196, 199-203. doi:10.1016/j.expneurol.2005.07.008

Radley, J.J., Rocher, A.B., Rodriguez, A., Ehlenberger, D.B., Dammann, M., McEwen, B.S., Morrison, J.H., Wearne, S.L., Hof, P.R., 2008. Repeated stress alters dendritic spine morphology in the rat medial prefrontal cortex. J. Comp. Neurol. 507, 1141-1150. doi: $10.1002 /$ cne. 21588

Radley, J.J., Sisti, H.M., Hao, J., Rocher, A., McCall, T., Hof, P., McEwen, B., Morrison, J., 2004. Chronic behavioral stress induces apical dendritic reorganization in pyramidal neurons of the medial prefrontal cortex. Neuroscience 125, 1-6. doi:10.1016/j.neuroscience.2004.01.006

Rauch, S.L., Shin, L.M., Segal, E., Pitman, R.K., Carson, M.A., McMullin, K., Whalen, P.J., Makris, N., 2003. Selectively reduced regional cortical volumes in post-traumatic stress disorder. Neuroreport 14, 913-916. doi:10.1097/01.wnr.0000071767.24455.10

Rockland, L., 1989. Psychoanalytically oriented supportive therapy: literature review and techniques. J Am Acad Psychoanal 17, 451-462.

Rogers, M. A., Yamasue, H., Abe, O., Yamada, H., Ohtani, T., Iwanami, A., Aoki, S., Kato, N., Kasai, K., 2009. Smaller amygdala volume and reduced anterior cingulate gray matter density associated with history of post-traumatic stress disorder. Psychiatry Res. 174, 210-216. doi:10.1016/j.pscychresns.2009.06.001

Roozendaal, B., McEwen, B.S., Chattarji, S., 2009. Stress, memory and the amygdala. Nat. Rev. Neurosci. 10, 423-433. doi:10.1038/nrn2651 


\section{ACCEPTED MANUSCRIPT}

Shapiro, F., 1989. Eye movement desensitization: a new treatment for post-traumatic stress disorder. J. Behav. Ther. Exp. Psychiatry 20, 211-217.

Shapiro, F., 2002. EMDR 12 years after its introduction: past and future research. J. Clin. Psychology 58, 1-22.

Shin, L.M., Liberzon, I., 2010. The neurocircuitry of fear, stress, and anxiety disorders. Neuropsychopharmacology 35, 169-191. doi:10.1038/npp.2009.83

Tzourio-Mazoyer, N., Landeau, B., Papathanassiou, D., Crivello, F., Etard, O., Delcroix, N., Mazoyer, B., Joliot, M., 2002. Automated anatomical labeling of activations in SPM using a macroscopic anatomical parcellation of the MNI MRI single-subject brain. Neuroimage 15, 273-289.

Ursano, R.J., Bell, C., Eth, S., Friedman, M., Norwood, A., Pfefferbaum, B., Pynoos, J.D.R.S., Zatzick, D.F., Benedek, D.M., McIntyre, J.S., Charles, S.C., Altshuler, K., Cook, I., Cross, C.D., Mellman, L., Moench, L.A., Norquist, G., Twemlow, S.W., Woods, S., Yager, J., Work Group on ASD and PTSD, Steering Committee on Practice Guidelines, 2004. Practice guideline for the treatment of patients with acute stress disorder and posttraumatic stress disorder. Am. J. Psychiatry 161, 3-31.

Ventureyra, V.A.G., Yao, S.-N., Cottraux, J., Note, I., De Mey-Guillard, C., 2002. The validation of the Posttraumatic Stress Disorder Checklist Scale in posttraumatic stress disorder and nonclinical subjects. Psychother. Psychosom. 71, 47-53.

Vermetten, E., Vythilingam, M., Southwick, S.M., Charney, D.S., Bremner, J.D., 2003. Longterm treatment with paroxetine increases verbal declarative memory and hippocampal volume in posttraumatic stress disorder. Biol. Psychiatry 54, 693-702. doi:10.1016/S0006-3223(03)00634-6

Vyas, A., Bernal, S., Chattarji, S., 2003. Effects of chronic stress on dendritic arborization in the central and extended amygdala. Brain Res. 965, 290-294.

Vyas, A., Pillai, A.G., Chattarji, S., 2004. Recovery after chronic stress fails to reverse amygdaloid neuronal hypertrophy and enhanced anxiety-like behavior. Neuroscience 128, 667-673. doi:10.1016/j.neuroscience.2004.07.013

Wignall, E.L., Dickson, J.M., Vaughan, P., Farrow, T.F.D., Wilkinson, I.D., Hunter, M.D., Woodruff, P.W.R., 2004. Smaller hippocampal volume in patients with recent-onset posttraumatic stress disorder. Biol. Psychiatry 56, 832-836. doi:10.1016/j.biopsych.2004.09.015

Yamasue, H., Kasai, K., Iwanami, A., Ohtani, T., Yamada, H., Abe, O., Kuroki, N., Fukuda, R., Tochigi, M., Furukawa, S., Sadamatsu, M., Sasaki, T., Aoki, S., Ohtomo, K., Asukai, N., Kato, N., 2003. Voxel-based analysis of MRI reveals anterior cingulate gray-matter volume reduction in posttraumatic stress disorder due to terrorism. Proc. Natl. Acad. Sci. U. S. A. 100, 9039-9043. doi:10.1073/pnas.1530467100 


\section{ACCEPTED MANUSCRIPT}

\section{Highlights:}

- This study explores the link between PTSD symptoms decrease and grey matter density increase in the prefrontal cortex.

- GM density enhancement in prefrontal areas parallels existing data in animals; thereby suggesting a potential reversibility of stress-induced effects in the prefrontal cortex.

- On the therapeutic edge, EMDR seems to be an efficient therapy to reduce PTSD symptoms and further restore brain structural organization.
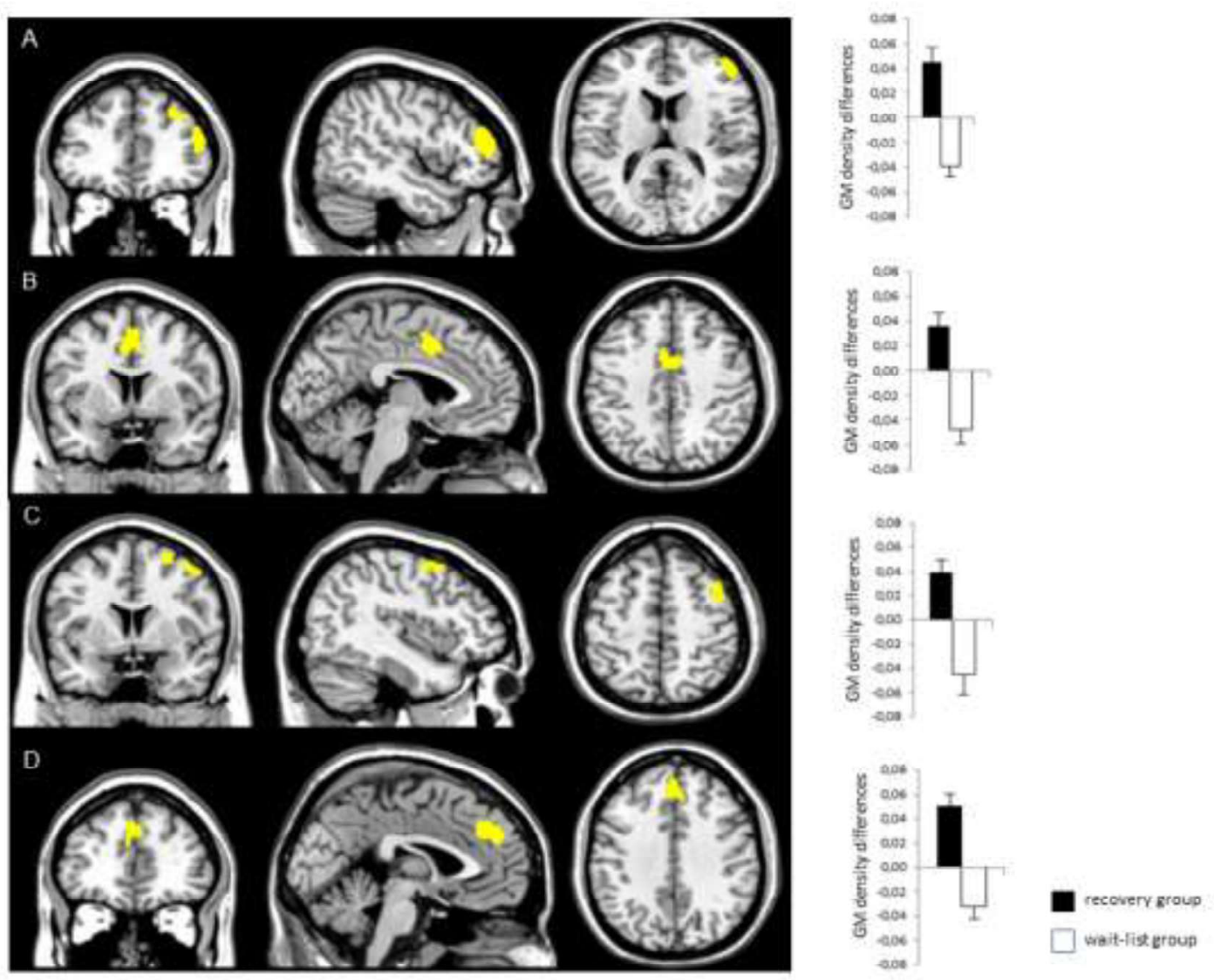